\title{
Changes in the normal appearing brain tissue and cognitive impairment in multiple sclerosis
}

\author{
M Filippi, C Tortorella, M Rovaris, M Bozzali, F Possa, M P Sormani, G Iannucci, \\ G Comi
}

\begin{abstract}
Objectives-To assess (a) whether the changes in the normal appearing brain tissue (NABT), as revealed by magnetisation transfer (MT) histogram analysis, correlates with cognitive dysfunction in patients with multiple sclerosis and (b) the relative contribution of these changes by comparison with that of multiple sclerosis lesions visible on conventional MRI.

Methods-Dual echo, T1 weighted and MT scans of the brain were obtained in 12 patients with multiple sclerosis with cognitive impairment and in seven without cognitive impairment. Lesion loads were assessed from $\mathrm{T} 2$ and $\mathrm{T} 1$ weighted scans. To create MT histograms of the NABT, multiple sclerosis lesion outlines from dual echo scans were superimposed automatically and nulled out from the coregistered and scalp stripped MTR maps. Average lesion MT ratio (MTR) and brain size were also measured.

Results-T2 and T1 lesion loads were significantly higher and the average lesion MTR and brain size were significantly lower in the group of cognitively impaired patients. Patients with cognitive deficits also had significantly lower average MTR and peak location of the NABT histogram. Logistic regression analysis showed that $68 \%$ of the total variance was explained by average NABT-MTR alone. A multivariable regression model showed that NABTMTR was the only factor that significantly correlated with cognitive impairment in these patients $(p=0.001)$.

Conclusions-The extent of abnormalities which go undetected when using conventional MRI is relevant in determining cognitive impairment in multiple sclerosis. (F Neurol Neurosurg Psychiatry 2000;68:157-161)
\end{abstract}

Keywords: multiple sclerosis; magnetic resonance imaging; magnetisation transfer imaging

Several studies have shown that in patients with multiple sclerosis, the extent of white matter abnormalities visible on conventional brain MRI, ${ }^{1-8}$ their regional distribution, ${ }^{238}$ and their intrinsic nature, as assessed on $\mathrm{T} 1$ weighted and magnetisation transfer imaging (MTI), ${ }^{67}$ all correlate well with cognitive impairment. In general, the degree of such correlations is higher than that found with physical disability. ${ }^{267}$
Lesion load estimates from conventional MRI are known not to give a complete picture of the burden of disease. ${ }^{9-11}$ Pathological studies showed that abnormalities can be detected in the brain tissue outside macroscopic multiple sclerosis lesions. ${ }^{12-16}$ These abnormalities include diffuse astrocytic hyperplasia, patchy oedema, perivascular cellular infiltration, and abnormally thin myelin and axonal damage. ${ }^{12-16}$ Such pathological abnormalities modify the relative proportions of mobile and immobile protons of the diseased tissue and, therefore, it is not surprising that MTI is able to show microscopic damage outside multiple sclerosis lesions visible on conventional T2 weighted scans. ${ }^{9-11}$ MT histogram analysis, as introduced by van Buchem et $a l^{17}$ allows evaluation of data from all the MR pixels of the brain tissue, thus providing a complete assessment of macroscopic and microscopic disease burden in multiple sclerosis. Measures derived from MT histograms from patients with multiple sclerosis are different from those from healthy controls, ${ }^{6}{ }^{71-19}$ and are correlated with clinical disability. ${ }^{7}{ }^{17-19}$ More recently, two studies showed that the overall disease burden (macroscopic lesions and subtle abnormalities in the normal appearing brain tissue (NABT)), as assessed by MT histograms of the entire brain, is correlated with the presence and severity of cognitive impairment in patients with multiple sclerosis. ${ }^{67}$ As in these studies the whole brain tissue was used to create MT histograms, the relative contributions of macroscopic lesions and of subtle NABT abnormalities to the cognitive decline in multiple sclerosis were not assessed. In the present study, we created MT histograms of the NABT to answer the following questions: (a) Is the damage of the NABT, as shown by the MT histogram analysis, relevant in determining cognitive dysfunction in patients with multiple sclerosis? and (b) If so, which is the relative contribution of these changes in comparison to that of multiple sclerosis lesions visible on conventional MRI?

Patients and methods

PATIENTS

We studied 19 patients (eight men and 11 women). Of these, 12 patients had cognitive impairment and seven had no evidence of cognitive impairment (see below). Patients were similar in age, level of education, disease duration, and neurological disability, as assessed by the expanded disability status scale (EDSS) (table 1). ${ }^{20}$ According to the Lublin and Reingold criteria, ${ }^{21} 11$ patients (four without and 
Table 1 Main demographic and clinical data from patients with multiple sclerosis with and without cognitive impairment

\begin{tabular}{llll}
\hline & Unimpaired & Impaired & pValue \\
\hline Mean age (SD) & $37.7(8.6)$ & $37.6(7.0)$ & NS \\
Mean duration of the education (SD) (y) & $11.6(3.8)$ & $11.1(4.2)$ & NS \\
Median disease duration (range) (y) & $15(6-20)$ & $11(2-18)$ & NS \\
Median EDSS (range) & $4.5(3.0-7.0)$ & $4.5(2.0-6.5)$ & NS
\end{tabular}

For statistical analysis, see text.

seven with cognitive impairment) had secondary progressive multiple sclerosis and eight (three without and five with cognitive impairment) had relapsing-remitting disease. At the time MRI was performed, patients were not in clinical relapse, nor were treated with steroids or psychoactive drugs in the 3 months before the study. All patients were unaffected by visual deficits or upper limb impairment that could interfere with their performance on the neuropsychological tests and had no history of drug or alcohol misuse or the presence of depression as assessed from clinical interviews before study entry. All patients signed a written informed consent before entering the study. The study design was approved by the local ethics committee.

\section{NEUROPSYCHOLOGICAL TESTS}

Neuropsychological tests exploring the executive functions (Weigl test, Dual task test, Wisconsin card sorting test (WCST), Stroop Colour/word interference test (Stroop test), Hanoi Towers test, verbal fluency test) and verbal and spatial memory (digit span test, short tale test, and Corsi span and supraspan test) were always obtained within 48 hours from the acquisition of the MRI. These tests are routinely used in the assessment of cognitive impairment in multiple sclerosis and are extensively described elsewhere. ${ }^{62-29} \mathrm{~Pa}$ tients' results on the Weigl test, verbal fluency test, digit span test, short tale test, and Corsi span and supraspan test were considered abnormal according to previously published criteria based on normative values obtained from a large group of Italian speaking subjects. ${ }^{30}$ For the other tests, patient performances were considered abnormal when they were equal to or above the 90th percentile (Stroop test, Hanoi towers test) and equal to or below the 5th percentile (WCST, dual task test) of the results obtained from a group of 20 sex and age matched healthy controls. Patients who had normal results in tests exploring the two cognitive domains (executive and memory functions) were classified as cognitively unimpaired, the others (patients with deficits of the executive functions, memory, or both) were classified as cognitively impaired. For each patient, the results from all neuropsychological tests were then scored, using a standardised method $^{30}$ based on a comparison with the percentile distribution of values from normal controls. These scores ranged from 0 to 4 , where grade 0 means a very poor performance and grade 4 means a normal performance. Individual test scores were then summed to provide a composite cognitive score for each patient.
IMAGE ACQUISITION

On a 1.5 Tesla scanner, each patient underwent dual echo turbo spin echo (TSE) $(T R=3300$, $\mathrm{TE}=16 / 98$, echo train length $(\mathrm{ETL})=6)$ scans. Twenty four axial $5 \mathrm{~mm}$ thick contiguous interleaved slices were obtained, with rectangular $188 \times 250 \mathrm{~mm}$ field of view and $194 \times 256$ image matrix, thus obtaining approximately a $1 \times 1 \mathrm{~mm}$ inplane resolution. MTI and T1 weighted magnetisation prepared rapid acquisition gradient echo (MP-RAGE) scans were also obtained in the same session. For MTI, 2D gradient echo images $(\mathrm{TR}=600, \mathrm{TE}=12$, flip angle $=20^{\circ}$ ) with and without a saturation pulse were obtained. The saturation pulse was an off resonance gaussian radiofrequency pulse centred $1.5 \mathrm{kHz}$ below the water frequency, with a duration of $16.4 \mathrm{~ms}$, a bandwidth of $250 \mathrm{~Hz}$, and a power intensity of $3.4 \times 10^{-6}$ Tesla. The same acquisition parameters as for the TSE scans were used for MTI scans, except for the number of slices, which was 20 . The set of slices for MTI was positioned to obtain the same central 20 slices as for the other acquisition schemes. From the two sets of imagesthat is, without (Mo) and with (Ms) saturation pulse and after their coregistration (see next paragraph)—quantitative MT ratio (MTR) images were derived pixel by pixel according to the following equation: $\mathrm{MTR}=(\mathrm{Mo}-\mathrm{Ms})$ / Mo $\times 100$, where Mo is the mean signal intensity for a given pixel without the saturation pulse and Ms is the mean signal intensity for the same pixel when the saturation pulse is applied. Signal intensities in the calculated images represented the MTR value. For MP-RAGE scans $(\mathrm{TR}=10, \mathrm{TE}=4, \mathrm{TI}=700$, flip angle $=10^{\circ}$, number of acquisitions $=1$ ), a 3D sagittal slab $(194 \times 256 \times 160$ image matrix, 250 $\mathrm{mm}$ field of view) covering the entire brain was acquired. This sequence gives the advantage that the acquired 3D images can be reconstructed in any plane and with different slice thicknesses and it has been shown that it enables a similar load of hypointense lesion to be detected as conventional T1 weighted scans do. ${ }^{31}$ The original data were then reformatted to obtain 24 axial contiguous $5 \mathrm{~mm}$ thick slices, with the same orientation and offsets as the corresponding TSE slices. Patients were positioned in the scanner using published guidelines for multiple sclerosis studies. ${ }^{32}$

IMAGE ANALYSIS

For the MRI data analysis, multiple sclerosis lesions were first outlined on proton density (PD) and MP-RAGE hard copies by the consensus of two experienced observers, who were unaware of the patients' clinical characteristics and cognitive test results. T2 weighted scans were always used to increase confidence in lesion detection. For T1 weighted MPRAGE scans, a conservative approach was used. Only areas with a signal intensity between those of the CSF and grey matter and with corresponding abnormalities on PD and T2 weighted images were considered as hypointense lesions. Lesion volume measurements were then performed by a trained technician, who was also unaware of patients' clinical 
Table $2 T 1$ and T2 lesion loads, average lesion MTR and brain volumes from patients with multiple sclerosis with and without cognitive impairment

\begin{tabular}{llll}
\hline & Unimpaired & Impaired & p Value \\
\hline Median T2 lesion load (range) (ml) & $8.4(2.4-18.4)$ & $38.4(4.7-73.6)$ & 0.002 \\
Median T1 lesion load (range) (ml) & $1.4(0.1-6.2)$ & $6.7(1.2-47.2)$ & 0.01 \\
T1/T2 ratio & $0.12(0.03-0.34)$ & $0.38(0.06-0.71)$ & 0.03 \\
Average lesion MTR (SD) (\%) & $35.1(2.0)$ & $32.1(3.0)$ & 0.006 \\
\hline
\end{tabular}

For statistical analysis, see text.

characteristics and cognitive test results. A highly reproducible local thresholding technique was used for lesion segmentation on computer displayed images, keeping the marked hard copies as a reference. ${ }^{33}$ Lesions were delineated as regions of interest (ROIs) and for each sequence the lesion volume was simply calculated by multiplying the total ROI area by the slice thickness. After image coregistration (see below), lesion outlines on PD weighted images were superimposed onto the MTR maps and average lesion MTR was calculated for each patient according to the following formula:

$$
\frac{\sum_{i=1}^{\mathrm{N}} \mathrm{A}_{i} \times \mathrm{MTR}_{i}}{\sum_{i=1 \quad \mathrm{~A}_{i}}^{\mathrm{N}}}
$$

where $\mathrm{N}$ is the number of lesions in a patient, $\mathrm{A} i$ is the area of lesion $i, \mathrm{MTR} i$ is the value of MTR in lesion $i$. Further details about this image analysis method are reported in detail elsewhere. ${ }^{33}$ Using the T1 weighted scans, parenchymal brain volumes were measured by a single observer using the same segmentation technique used for lesion volume measurements. ${ }^{33}$

From each MTI scan we also derived histograms of MTR values for the NABT, defined as the overall brain tissue not involved by lesions visible on dual echo scans (thus including both normal appearing white and grey matter). Firstly, we removed the skull and other extracranial tissues from the PD and the GE images without and with the MT pulse, with the same local thresholding technique used for lesion segmentation. ${ }^{33}$ Secondly, the scalp stripped GE images were coregistered and MTR maps obtained. Then, the MTR maps were coregistred with the corresponding scalp stripped PD weighted images. Coregistration of images was performed using a surface matching technique which fits the contours corresponding to the CSF dura interface of the two MR images. This technique estimates the spatial transformation needed to remap images from different MRI studies into the same spatial reference system, thus enabling a pixel by pixel correspondence between different MRI studies and ensuring the correct positioning of the ROI across multiple images. ${ }^{34}{ }^{35}$ The optimal transformation is inferred from the minimisation of the distance between the two discrete surfaces. Validation studies have shown that the accuracy of realignment was of the order of the image pixel size. ${ }^{35}$ Finally, the macroscopic lesions segmented on PD weighted images were superimposed automatically onto the coregistred MTR map, and the areas corresponding to the segmented lesions were nulled out. To reduce partial volume averaging from the CSF, we excluded from the analysis all the pixels with MTR values lower than $10 \%$. In this way only pixels belonging to macroscopically normal brain tissue remained. To correct for the between patient differences in brain volume, each histogram was normalised by dividing it by the total number of pixels included. For each MT histogram, we analysed the height and the location of the histogram peak with respect to the $\mathrm{x}$ axis (the MTR value most represented in the brain) and the average MTR value.

\section{STATISTICAL ANALYSIS}

Group differences between cognitively impaired and unimpaired patients were evaluated using the Student's $t$ test for non-paired data when the data were distributed normally and the Mann-Whitney test when the data were not normally distributed. To assess the factors that are independently associated with cognitive impairment we run a multivariable logistic regression model (forward selection analysis), using as explanatory variables $\mathrm{T} 1$ and $\mathrm{T} 2$ lesion loads, average lesion MTR, and NABT-MT histogram derived measures and brain size. The relative contributions of each of the above mentioned variables were calculated from the full logistic regression model. Univariate correlations between different MR measures and between MTR derived measures and the composite cognitive score were assessed using the Spearman rank correlation coefficient.

\section{Results}

The loads of multiple sclerosis lesions visible on T2 and T1 weighted scans, as well as their average MTR are reported in table 2. Both T2 and T1 lesion loads were significantly higher in the group of cognitively impaired patients. The ratio between $\mathrm{T} 1$ weighted and $\mathrm{T} 2$ weighted lesion loads was significantly higher and the average lesion MTR significantly lower in this patient group, thus indicating that these patients not only have more lesions but also a higher proportion of them have more severe tissue damage. Cognitively impaired patients also had slightly smaller mean brain volumes than the unimpaired patients (1203 (SD 85) $\mathrm{ml} v 1307$ (SD 57) $\mathrm{ml}$; $\mathrm{p}=0.03$ ).

The MT derived histogram parameters for the NABT are reported in table 3. The MT histograms from the NABT are presented in the figure for the two groups of patients. Patients with cognitive deficits had significantly lower average MTR and peak location. The average NABT-MTR correlated significantly

Table 3 MT histogram derived measures of the normal appearing brain tissue (NABT) from patients with multiple sclerosis with and without cognitive impairment

\begin{tabular}{lrlc}
\hline & Unimpaired & Impaired & p Value \\
\hline Average MTR (SD) (\%) & $41.6(0.5)$ & $39.8(0.9)$ & $<0.0001$ \\
Peak height & $103.0(9.1)$ & $92.5(12.7)$ & 0.07 \\
Peak location & $37.1(0.9)$ & $34.7(1.2)$ & $<0.0001$ \\
\hline
\end{tabular}

^Student's $t$ test for unpaired data. 


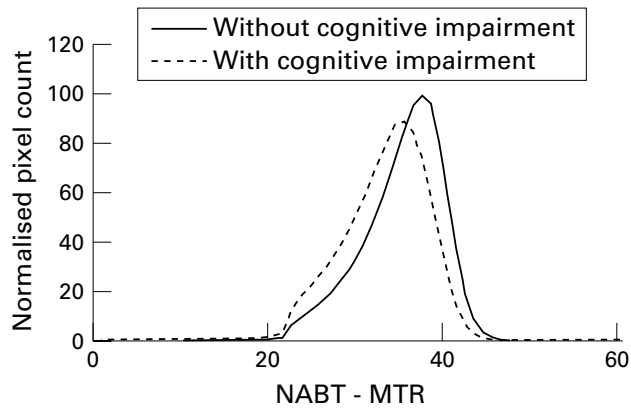

MT histograms of the normal appearing brain tissue (NABT) in patients with multiple sclerosis with and without cognitive impairment.

with the T2 $(r=-0.78, \mathrm{p}<0.0001)$ and the T1 $(r=-0.60, \mathrm{p}=0.006)$ lesion loads. The percentages of the total variance explained by each individual factor included in the model were as follows: average NABT-MTR $=68 \%$, $\mathrm{T} 1$ lesion load $=38 \%$, T2 lesion load $=37 \%$, average lesion MTR $=35 \%$, and brain size $=21 \%$. The multivariable regression model showed that average NABT-MTR $(p<0.001)$ was the only factor that was significantly correlated with cognitive impairment in our patients. The other tested variables ( $\mathrm{T} 1$ and $\mathrm{T} 2$ lesion loads, average lesion MTR, NABT histogram peak height and location, and brain size) were all removed from the model. Significant correlations were also found between the composite cognitive score and average lesion MTR $(r=0.72, \mathrm{p}<0.001)$, average NABT-MTR $(r=0.75, \mathrm{p}<0.0001)$, peak height of the NABT-MTR histogram $(r=0.65, \mathrm{p}=0.004)$, and peak location of the NABT-MTR histogram $(r=0.77, \mathrm{p}<0.0001)$.

\section{Discussion}

Conventional MRI is very sensitive in detecting multiple sclerosis lesions and their changes over time. ${ }^{87}$ For this reason MRI is widely used to monitor multiple sclerosis evolution, both natural or modified by treatment. ${ }^{37}$ However, it is known that conventional MR techniques, among other limitations, ${ }^{9}$ are not able to detect the full extent of changes seen pathologically in multiple sclerosis. ${ }^{9}$ These include subtle abnormalities in the NABT. ${ }^{-15}$ Although multiple sclerosis related changes in the NABT have to be subtle, otherwise they would result in visible areas of focal abnormalities on conventional scanning, they may be so extensive as to have some influence on the evolution of the disease. The following points support the concept that the extent of the microscopic changes in the NABT may contribute to the development of disability in multiple sclerosis. Firstly, the MTR of the white matter away from multiple sclerosis lesions and the MTR in the white matter around such lesions from patients with secondary progressive multiple sclerosis are lower than those of the corresponding regions from patients with less disabling relapsing-remitting multiple sclerosis. ${ }^{10} 39$ Secondly, white matter outside macroscopic multiple sclerosis lesions from patients with secondary progressive multiple sclerosis has an $\mathrm{N}$-acetyl aspartate to creatine ratio on average $8.2 \%$ lower than the that from patients with relapsing-remitting multiple sclerosis. ${ }^{40}$ In patients with relapsingremitting multiple sclerosis a progressive reduction of this ratio is detectable over time and its decrease correlates strongly with changes in physical disability. ${ }^{40}$ Although changes in the brain tissue which appears normal on conventional scanning are more likely to have an impact on cognitive functioning of multiple sclerosis patients rather than on physical disability, no previous study has assessed the contribution of such changes to the impairment of cognition.

The present study confirms that the extent of the lesions seen on conventional MRI and the severity of tissue disorganisation within these lesions are relevant in determining cognitive impairment in patients with multiple sclerosis. ${ }^{1-8}$ This study also clearly shows that NABT changes do make a significant contribution to multiple sclerosis clinical manifestations. Clearly, MTI is not the only MR technique that detects such subtle changes in the normal appearing white matter of the patients we studied. Therefore, it is likely that other quantitative MR techniques, such as the analysis of $\mathrm{T} 1$ and $\mathrm{T} 2$ relaxation times, diffusion measurements and imaging, and MR spectroscopy, might also provide useful information. That widespread NABT damage is relevant for cognitive decline in multiple sclerosis is in agreement with the results of previous PET studies, ${ }^{41}{ }^{42}$ suggesting that cognitive decline in multiple sclerosis may be the result of widespread damage to deep brain structures, leading to functional disconnection of different cortical areas. This finding also agrees with the relatively low correlations found by previous studies between regional MR abnormalities and deficits in specific cognitive domains. ${ }^{56}$ The recent demonstration that significant axonal loss occurs in the white matter outside macroscopic multiple sclerosis lesions provides further evidence that changes which go undetected on conventional scanning may be functionally relevant. ${ }^{15} 16$

Because normal appearing white matter represents a large part of the tissue included in NABT MT histograms, we think that microscopic white matter abnormalities were the major contributing factor to the observed differences between the MT histograms of the two groups of patients. Nevertheless, it is likely that lesions in or adjacent to the cerebral cortex, which can be imaged using fast fluid attenuated inversion recovery sequences, ${ }^{43}{ }^{44}$ may have been missed in the present study and may also have contributed to the MT histogram changes seen for NABT and, as a consequence, to cognitive impairment. ${ }^{8}$ The role of pathological changes in the basal ganglia is likely to be minor, if present at all, due to the low frequency of clinical or MRI involvement of these structures in multiple sclerosis. ${ }^{45}{ }^{46} \mathrm{We}$ can only speculate about the nature of the subtle changes potentially responsible for the cognitive impairment seen in our patients. Small focal abnormalities independent of larger lesions visible on conventional scans or damage to axons transversing large focal lesions and 
resulting in wallerian degeneration in areas away from such abnormalities may both contribute to cognitive impairment. ${ }^{47-51}$ The results of the present study allow us to propose that these abnormalities are not mutually exclusive. The larger amount of macroscopic lesions seen in cognitively impaired patients and the correlations found between NABT changes and $\mathrm{T} 1$ and $\mathrm{T} 2$ lesion loads suggest the role of secondary axonal degeneration. However, the larger contribution of NABTMTR than lesion extent and severity to the presence of cognitive impairment, as shown by the multivariable analysis, suggests that subtle focal abnormalities other than secondary axonal degeneration are also relevant.

This study was supported by a grant from the Istituto Superiore di Sanità. MPS is supported by a grant from Teva Italy. We are grateful to Dr J C Sipe for his helpful manuscript revision, Drs M Falautano, V Martinelli, and G Santuccio who assisted us in the recruiment of the patients, and Drs L Minicucci, C Pereira, and G Rizzo for their technical assistance.

1 Rao SM, Leo GJ, Haughton VM, et al. Correlation of magnetic resonance imaging with neuropsychological testing in multiple sclerosis. Neurology 1989;39:161-6.

2 Swirsky-Sacchetti T, Mitchell DR, Seward J, et al. Neuropsychological and structural brain lesions in multiple sclerosis: a regional analysis. Neurology 1992;42:1291-5.

3 Arnett PA, Rao SM, Bernardin L, et al. Relationship between frontal lobe lesions and Wisconsin card sorting test performance in patients with multiple sclerosis. Neurology $1994 ; 44: 420-5$.

4 Comi G, Filippi M, Martinelli V, et al. Brain MRI correlates of cognitive impairment in primary and secondary progresof cognitive impairment in primary and secondary prog
sive multiple sclerosis. $\mathcal{F}$ Neurol Sci 1995;132:222-7.

5 sive multiple sclerosis. F Neurol Sci 1995;132:222-7. tions in multiple sclerosis. The role of frontal lobe patholtions in multiple sclerosis. The
ogy. Brain 1997;120:15-26.

6 Rovaris M, Filippi M, Falautano M, et al. Relation between MR abnormalities and patterns of cognitive impairment in multiple sclerosis. Neurology 1998;50:1601-8.

7 van Buchem MA, Grossman RI, Armstrong C, et al. Correlation of volumetric magnetization transfer imaging with clinical data in MS. Neurology 1998;50:1609-17.

8 Miki Y, Grossman RI, Udupa JK, et al. Isolated U-fiber involvement in MS. Preliminary observations. Neurology 1998;50:1301-6.

9 Filippi $M$. The role of non-conventional magnetic resonance techniques in monitoring the evolution of multiple sclerosis. I Neurol Neurosurg Psychiatry 1998;118(suppl 1): $1601-12$.

10 Filippi M, Campi A, Dousset V, et al. A magnetization transfer imaging study of normal-appearing white matter in transfer imaging study of normal-appearing whit
multiple sclerosis. Neurology 1995;45:478-82.

11 Loevner LA, Grossman RI, Cohen JA, et al. Microscopic disease in normal-appearing white matter on conventional disease in normal-appearing white matter on conventional
images in patients with multiple sclerosis: assessment with images in patients with multiple sclerosis: assessment with 511-15.

12 Adams CMW. Pathology of multiple sclerosis: progression of the lesion. Br Med Bull 1977;33:15-20.

13 Allen IV, McKeown SR. A histological, histochemical and biochemical study of the macroscopically normal whit matter in multiple sclerosis. F Neurol Sci 1979;41:81-91.

14 Arstila AU, Riekkinen P, Rinne UK, et al. Studies on the pathogenesis of multiple sclerosis. Participation of lysosomes on demyelination in the central nervous system white matter outside plaques. Eur Neurol 1973;9:1-20.

15 Trapp BD, Peterson J, Ransohoff, et al. Axonal transection in the lesions of multiple sclerosis. N Eng f Med 1998;338: in the lesior 278 .

16 Evangelou N, Esiri MM, Palace J, et al. A quantitative pathological study of axonal loss in the corpus callosum in
multiple sclerosis [abstract]. Multiple Sclerosis 1998;4:287.

17 van Buchem MA, McGowan JC, Kolson DL, et al. Quantitative volumetric magnetization transfer analysis in multitative volumetric magnetization transfer analysis in multi-
ple sclerosis: estimation of macroscopic and microscopic ple sclerosis: estimation of macroscopic and micicos
disease burden. Magn Reson Med 1996;36:632-6.

18 Filippi M, Iannucci G, Tortorella C, et al. Comparison of MS clinical phenotypes using conventional and magnetization transfer MRI. Neurology 1999;52:588-94.

19 Mastronardo G, Iannucci G, Rocca MA, et al. Correlations between clinical and MRI cerebellar involvement in multiple sclerosis: assessment with T2, T1 and MT histogram [abstract]. Neurology 1998;50(suppl 4):108.

20 Kurtzke JF. Rating neurological impairment in multiple sclerosis: an expanded disability status scale (EDSS). Neurology 1983;33:1444-52.

21 Lublin FD, Reingold SC, the National Multiple Sclerosis Society (USA) Advisory Committee on Clinical Trials of Society (USA) Advisory Committee on Clinical Trials of
New Agents in Multiple Sclerosis. Defining the clinical course of multiple sclerosis: results of an international surcourse of multiple sclerosis: results
vey. Neurology 1996;46:907-11.
22 Peyser JM, Edwards KR, Poser CM, et al. Cognitive function in patients with multiple sclerosis. Arch Neurol 1980;37:577-9.

23 Badeley A, Della Sala S, Papagno C, et al. Dual task performance in dysexecutive and non-dysexecutive patient with a frontal lesion. Neuropsychology 1997;11:187-94.

24 Weigl E. On the psychology of the so called processes of abstraction. F Abnorm Soc Psychol 1927;36:3-33.

25 Heaton RH. Wisconsin card sorting test (manual). Odessa, FL: Psychological Assessment Resources, 1981.

26 Stroop JR. Studies of interference in serial verbal reactions. f Exp Psychol 1935;18:643-62.

27 Pozzilli C, Bastianello S, Padovani A, et al. Anterior corpus callosum atrophy and verbal fluency in multiple sclerosis. Cortex 1991;27:441-5.

28 Shallice T. Specific impairments of planning. Philos Trans $R$ Soc Lond B Biol Sci 1982;298:199-209.

29 Pagano G, Valla RG. Verbal and special immediate memory span. Normative data from 1355 adults and 1112 children. Ital f Neurol Sci 1987;8:539-48.

30 Spinnler H, Tognoni G. Standardizzazione e taratura italiana di test neuropsicologici. It $\mathcal{F}$ Neurol Sci 1987; (suppl 6).

31 Filippi M, Rocca MA, Horsfield MA, et al. Increased spatial resolution using a three-dimensional T1-weighted gradient-echo MR sequence results in greater hypointense lesion volumes in multiple sclerosis. A fNR Am $\mathscr{f}$ Neuroradiol 1998;19:235-8.

32 Miller DH, Barkhof F, Berry I, et al. Magnetic resonance imaging in monitoring the treatment of multiple sclerosis: concerted action guidelines. I Neurol Neurosurg Psychiatry 1991;54:683-8.

33 Rovaris M, Filippi M, Calori G, et al. Intra-observer reproducibility in measuring new MR putative markers of demyelination and axonal loss in multiple sclerosis: a comparison with conventional T2-weighted images. F Neurol 1997; 244:266-70.

34 Robb RA, Hanson DP, Karwoski RA, et al. Analyze: a comprehensive operator-interactive software package for multidimensional medical image display and analysis. Comp Med Imag Graph 1989;13:433-54.

35 Jiang H, Robb R, Holton KS. A new approach to 3-D registration of multimodality medical images by surface matching. Proc SPIE 1992;1808:196-213.

36 West J, Fitzpatrick JM, Wang MY, et al. Comparison and evaluation of retrospective intermodality brain image regisevaluation of retrospective intermodality brain image regis-
tration techniques. 7 Comput Assist Tomogr 1997;21:55466.

37 Miller DH, Albert PS, Barkhof F, et al. Guidelines for the use of magnetic resonance techniques in monitoring the treatment of multiple sclerosis. Ann Neurol 1996;39:6-16.

38 Filippi M, Horsfield MA, Ader H, et al. Guidelines for using quantitative measures of brain magnetic resonance imaging abnormalities in monitoring the treatment of multiple sclerosis. Ann Neurol 1998;43:449-506.

39 Dousset V, Grossman RI, Ramer KN, et al. Experimental allergic encephalomyelitis and multiple sclerosis: lesion characterization with magnetization transfer imaging. Radiology 1992;182:483-91.

$40 \mathrm{Fu} \mathrm{L}$, Matthews PM, De Stefano N, et al. Imaging axonal damage of normal-appearing white matter in multiple sclerosis. Brain 1998;121:103-13.

41 Paulesu E, Perani D, Fazio F, et al. Functional basis of memory impairment in multiple sclerosis: a $\left[{ }^{18} \mathrm{~F}\right] \mathrm{FDG}$ PET study. Neuroimage 1996;4:87-96.

42 Brooks DJ, Leenders KL, Head G, et al. Studies on regional cerebral oxygen utilisation and cognitive function in multiple sclerosis. F Neurol Neurosurg Psychiatry 1984;47:118291.

43 Filippi M, Yousry TA, Baratti C, et al. Quantitative assessment of MRI lesion load in multiple sclerosis. A comparison of conventional spin-echo with fast fluidattenuated inversion recovery. Brain 1996;119:1349-55.

44 Gawne-Cain ML, O'Riordan JI, Thompson AJ, et al. Multiple sclerosis lesion detection in the brain: a comparison of fast fluid-attenuated inversion recovery and conventional T2-weighted dual spin-echo. Neurology 1997;49:364-70.

45 McAlpine D, Lumsden CE, Acheson ED. Multiple sclerosis: a reappraisal. Baltimore: Williams and Wilkins, 1972:177-8.

6 Grimaud J, Millar J, Thorpe JW, et al. Signal intensity on MRI of basal ganglia in multiple sclerosis. F Neurol Neurosurg Psychiatry 1995;59:306-8.

47 Barbosa S, Blumhardt LD, Roberts N, et al. Magnetic resonance relaxation time mapping in multiple sclerosis: normal appearing white matter and the "invisible" lesion load. Magn Reson Med 1994;12:33-42.

48 Filippi M, Horsfield MA, Campi A, et al. Resolutiondependent estimates of lesion volumes in magnetic resonance imaging studies of the brain in multiple sclerosis Ann Neurol 1995;38:749-54.

49 Keiper MD, Grossman RI, Hirsch JA, et al. MR identification of white matter abnormalities in multiple sclerosis : a comparison between $1.5 \mathrm{~T}$ and $4 \mathrm{~T}$. AfNR Am f Neuroradiol 1998;19:1489-93.

50 De Stefano N, Narayanan S, Matthews PM, et al. In vivo evidence for axonal dysfunction remote from focal cerebral demyelination of the type seen in multiple sclerosis. Brain 1999;122:1933-9.

51 De Stefano N, Mathhews PM, Narayanan S, et al. Axonal dysfunction and disability in a relapse of multiple sclerosis: longitudinal study of a patient. Neurology 1997;49:113841 . 